\title{
Identification and characterization of RET fusions in advanced colorectal cancer
}

\author{
Anne-France Le Rolle ${ }^{1,2, *}$, Samuel J. Klempner ${ }^{1,2, *}$, Christopher R. Garrett ${ }^{3}$, Tara \\ Seery ${ }^{1,2}$, Eric M. Sanford ${ }^{4}$, Sohail Balasubramanian ${ }^{4}$, Jeffrey S. Ross ${ }^{4,5}$, Philip J. \\ Stephens ${ }^{4}$, Vincent A. Miller ${ }^{4}$, Siraj M. Ali ${ }^{4}$ and Vi K. Chiu ${ }^{1,2}$ \\ ${ }^{1}$ Division of Hematology/Oncology, Department of Medicine, University of California Irvine, Irvine, CA, USA \\ ${ }^{2}$ Chao Family Comprehensive Cancer Center, University of California Irvine, Orange, CA, USA \\ ${ }^{3}$ The Division of Cancer Medicine, Department of Gastrointestinal Medical Oncology, MD Anderson Cancer Center, Houston, \\ TX, USA \\ ${ }^{4}$ Foundation Medicine Inc., Cambridge, MA, USA \\ ${ }^{5}$ Albany Medical College, Albany, NY, USA \\ * These authors have contributed equally to this work \\ Correspondence to: Vi K. Chiv, email: vkchiu@uci.edu \\ Keywords: RET fusion kinase, RET kinase inhibitor, comprehensive genomic profiling, colorectal cancer \\ Received: April 02, $2015 \quad$ Accepted: May 12, $2015 \quad$ Published: May 30, 2015
}

This is an open-access article distributed under the terms of the Creative Commons Attribution License, which permits unrestricted use, distribution, and reproduction in any medium, provided the original author and source are credited.

\section{ABSTRACT}

There is an unmet clinical need for molecularly directed therapies available for metastatic colorectal cancer. Comprehensive genomic profiling has the potential to identify actionable genomic alterations in colorectal cancer. Through comprehensive genomic profiling we prospectively identified 6 RET fusion kinases, including two novel fusions of CCDC6-RET and NCOA4-RET, in metastatic colorectal cancer (CRC) patients. RET fusion kinases represent a novel class of oncogenic driver in CRC and occurred at a $0.2 \%$ frequency without concurrent driver mutations, including KRAS, NRAS, BRAF, PIK3CA or other fusion tyrosine kinases. Multiple RET kinase inhibitors were cytotoxic to RET fusion kinase positive cancer cells and not RET fusion kinase negative CRC cells. The presence of a RET fusion kinase may identify a subset of metastatic CRC patients with a high response rate to RET kinase inhibition. This is the first characterization of RET fusions in CRC patients and highlights the therapeutic significance of prospective comprehensive genomic profiling in advanced CRC.

\section{INTRODUCTION}

Colorectal cancer (CRC) is the second most common cause of cancer-related death in the United States. Currently, metastatic CRC patients are treated mainly as an unselected cohort with angiogenesis inhibitors and cytotoxic chemotherapy. Only the absence of an oncogenic $K R A S$ or NRAS mutation has been found to predict clinical benefit from treatment with anti-EGFR antibodies [1]. Although recent molecular characterization of CRC has not yet been translated into effective therapeutic strategies [2], comprehensive genomic profiling has emerged as a promising approach that enables the identification of genomic biomarkers that may inform the use of targeted therapy in clinical trials. This therapeutic genomic paradigm is best demonstrated in tumors that are driven by activated protein tyrosine kinases due to oncogenic mutations or rearranged chromosomal fusion [3]. Classic examples include gefitinib inhibition of EGFR mutant kinase in non-small cell lung cancer (NSCLC) and imatinib inhibition of $B C R-A B L$ fusion kinase in chronic myeloid leukemia $[4,5]$.

Oncogenic RET point mutations and rearranged $R E T$ fusions induce hereditary and sporadic tumors [6] . $R E T$ fusion kinase occurs in nearly one-third of papillary thyroid cancer and $\sim 2 \%$ of lung adenocarcinoma, but is not yet identified in CRC [6]. RET fusion kinase juxtaposes the C-terminal RET kinase domain to an $\mathrm{N}$-terminal coiled-coil or leucine zipper dimerization domain from multiple 5 ' fusion partners to trigger ligand 
independent activation of downstream signaling pathways such as RAS-MAPK and PI3K-AKT [6, 7]. Here, we prospectively identified by comprehensive genomic profiling the presence of RET fusion kinase in CRC patients. Evidence of therapeutic response in CRC patient with a CCDC6-RET fusion treated with the RET kinase inhibitor regorafenib highlights the therapeutic importance of genomic profiling in colorectal cancer.

\section{RESULTS}

\section{Characterization of $R E T$ fusions in CRC patients}

To identify novel oncogenic drivers in colorectal cancer that may be targeted therapeutically, we performed prospective comprehensive genomic profiling using next generation sequencing (NGS) on metastatic colorectal tumors in the complete coding sequence of 236 cancerrelated genes and the introns of 19 frequently rearranged cancer-related genes (Supplemental Table 1). Prior retrospective analyses with NGS of 40 CRC specimens detected a $C 2$ orf 44-ALK fusion kinase but did not identify any $R E T$ fusion kinase [8]. As expected, we detected mutations in $K R A S, P I K 3 C A, B R A F, p 53$ and $A P C$, which are known mutated oncogenes and tumor suppressors in CRC (data not shown). We prospectively profiled 3,117 metastatic colorectal tumors, and identified the presence of 6 RET fusion kinases to give a frequency of $0.2 \%$ (Figure 1A). The clinicopathologic characteristics of these six RET fusion-positive CRC patients revealed the absence of a concurrent driver mutation or other fusion tyrosine kinases (Figure 1B). We identified two novel RET fusion

A

B

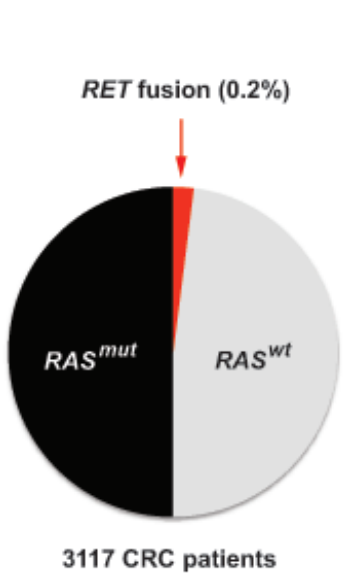

\begin{tabular}{cccc}
\hline Characteristics & Patient 1 & Patient 2 & Patient 3 - 6 \\
\hline Age & 63 & 53 & nd \\
Sex & Female & Male & nd \\
Race & Asian & nd & nd \\
Smoking Status & Never & Never & nd \\
Histology & AdenoCA & AdenoCA & AdenoCA \\
Tumor Grade & $2 / 3$ & $2 / 3$ & $2 / 3$ \\
Tumor Stage & IV & IV & IV \\
Primary Tumor Site & Sigmoid & Rectosigmoid & Colorectal \\
Prior Regimens & 0 & $>3$ & nd \\
MSI & Stable & Stable & Stable \\
KRAS \& NRAS & WT & WT & WT \\
BRAF & WT & WT & WT \\
PIK3CA & WT & WT & WT \\
ALK & WT & WT & WT \\
ROS1 & WT & WT & WT \\
NTRK1-3 & WT & WT & WT \\
\hline
\end{tabular}

C

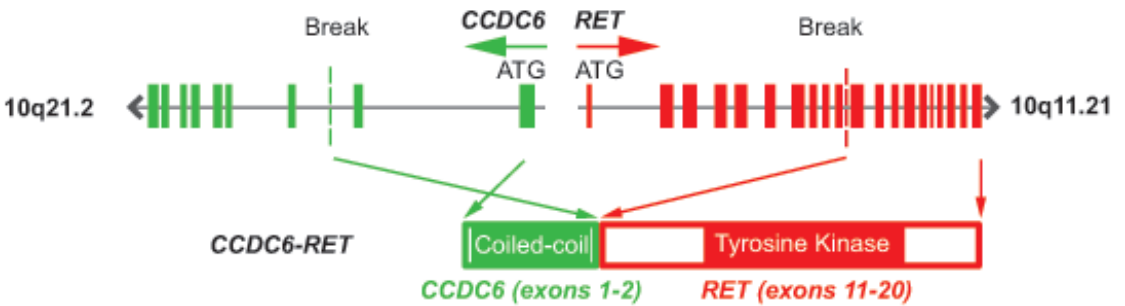

D

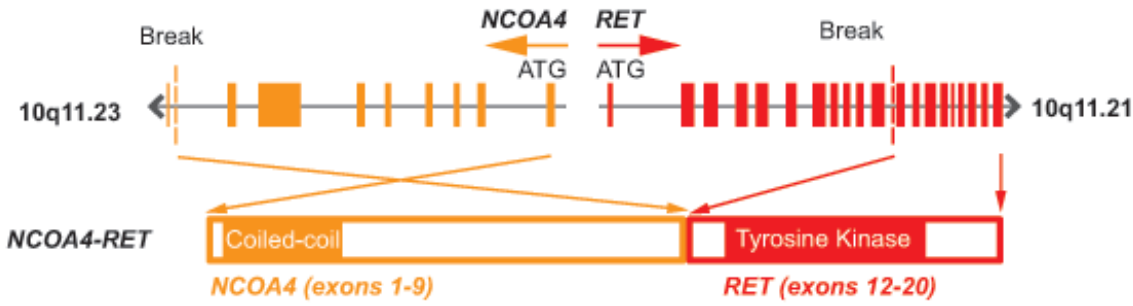

Figure 1: Characterization of $\boldsymbol{R E T}$ fusions in CRC patients. A. Frequency of $R E T$ fusions in unselected metastatic CRC patients as detected by NGS. B. Genetic and clinicopathologic characteristics of 6 patients harboring $R E T$ fusion kinase. nd $=$ no data and WT $=$ wild type. C. Fusion of CCDC6 exon 11 (green) containing the coiled-coil domain to RET exon 11 (red) containing the tyrosine kinase domain to generate CCDC6-RET fusion kinase. D. Fusion of $N C O A T$ exon 9 (orange) containing the coiled-coil domain to RET exon 12 (red) containing the tyrosine domain to generate NCOAT-RET fusion kinase. 
kinases involving 5' fusion partners Coiled Coil Domain Containing 6 (CCDC6) and Nuclear Receptor Coactivator 4 (NCOA4) in two patients with metastatic CRC. Patient 1 had a CCDC6-RET fusion kinase with amino-terminal $C C D C 6$ exon 1-2 and carboxyl-terminal RET exon 11-19 (Figure 1C). The CCDC6-RET fusion kinase in patient 1 occurred at a novel breakpoint at CCDC6 intron 2 and RET intron 10 from a chromosome 10 inversion event, which differs from the CCDC6-RET fusion kinase breakpoints in thyroid cancer and lung cancer that fused $C C D C 6$ exon
1 to RET exon 12-20 [9]. Patient 2 had an NCOA4-RET fusion kinase with amino-terminal $N C O A 4$ exons 1-9 and carboxyl-terminal RET exons 12-19, with breakpoints at NCOA4 intron 9 and RET intron 11 from a chromosome 10 tandem duplication event (Figure 1D). The $N C O A 4 \alpha$ isoform observed in patient 2 was nearly full length in comparison to the truncated NCOA4 $\beta$ isoform fused to $R E T$ exon 12 in papillary thyroid carcinoma and NSCLC adenocarcinoma $[10,11]$.
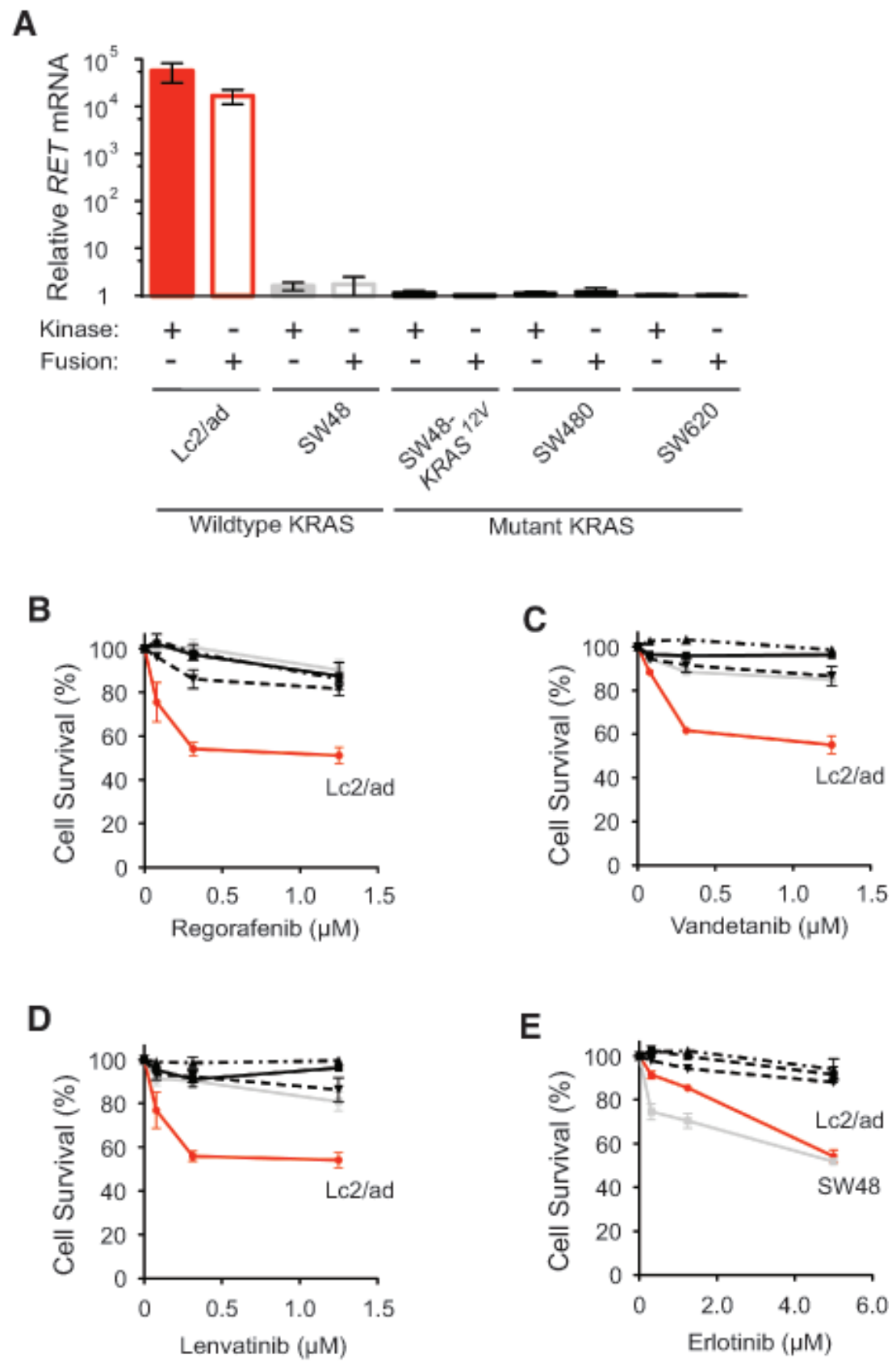

Figure 2: Inhibition of $\boldsymbol{R E T}$ fusion-positive cancer cells viability by RET kinase inhibitors. A. Relative $R E T$ mRNA levels in Lc2/ad, SW48, SW48,-KRAS ${ }^{12 \mathrm{~V}}$, SW480, and SW620 cells as measured by quantitative RT-PCR analysis and normalized to SW620 cells using primers that recognized the RET kinase domain (Kinase: + ) or flanked the CCDC6-RET fusion site (Fusion: +). B.-E. Lc2/ad, SW48, SW48,-KRAS ${ }^{12 \mathrm{~V}}$, SW480, and SW620 cells were treated with indicated concentrations of regorafenib B. vandetanib C. lenvatinib D. and erlotinib E. for 72 hours and cell survival was determined relative to $0.1 \%$ DMSO-treated controls $(\operatorname{mean} \pm \mathrm{STD} ; n=3)$. 


\section{Cytotoxic effect of RET kinase inhibitors in RET fusion-positive cancer cells}

Because regorafenib inhibits the proliferation of thyroid TT cells driven by oncogenic $R E T^{\mathrm{C} 634 \mathrm{~W}}$ point mutation [12], we next tested the ability of regorafenib to inhibit RET fusion-positive cancer cells. Using two different primer pairs that either bound within the RET kinase domain or flanked the CCDC6-RET fusion site, we confirmed by quantitative PCR that Lc2/ad cancer cells, but not several KRAS wildtype and mutant CRC cells, harbored the CCDC6-RET fusion kinase (Figure 2A) [13]. Treatment with nanomolar concentration of regorafenib was cytotoxic to Lc2/ad cells, but the RET fusion- negative $\mathrm{CRC}$ cells remained resistant even at micomolar concentration (Figure 2B). Vandetanib and lenvatinib, which are FDA-approved RET kinase inhibitors with clinical efficacy against RET fusion-positive thyroid cancer $[14,15]$, followed the same pattern and specifically inhibited only Lc2/ad cells viability (Figure 2C and 2D). In contrast, both KRAS wildtype Lc2/ad and SW48 cells had increased sensitivity to erlotinib treatment, and KRAS mutant CRC cells were resistant to this EGFR kinase inhibitor as predicted (Figure 2E) [16]. RET kinase inhibitors specifically suppressed only $R E T$ fusion-positive cancer cells viability and did not show non-specific suppression of RET fusion-negative CRC cells viability with either KRAS wildtype or mutant status.

A
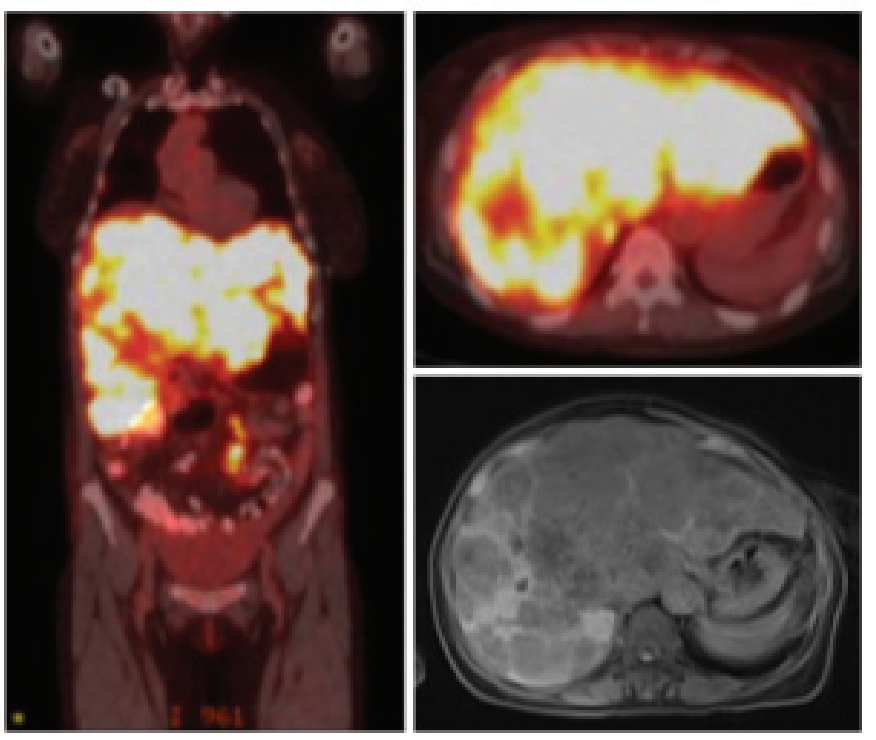

B

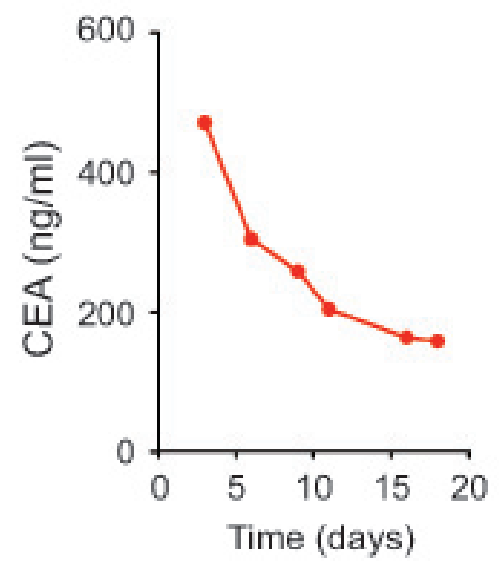

C

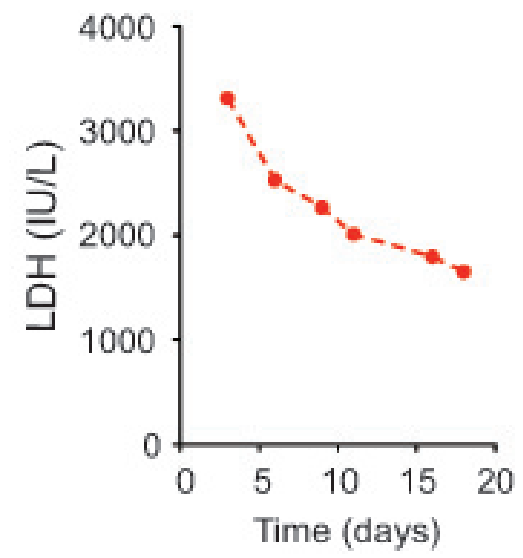

Figure 3: Clinical response of $\boldsymbol{R E T}$ fusion-positive CRC patient to regorafinib. A. Scans of Patient 1 harboring $C C D C 6-R E T$ fusion kinase with diffuse liver metastases evident on PET/CT scan on coronal (left) and transverse (upper right) sections and MRI scan on transverse section (lower right). B. Serum CEA of patient 1 treated with regorafenib 40-80 mg daily. C. Serum LDH of Patient 1 treated with regorafenib 40-80 mg daily. 


\section{Clinical response of $R E T$ fusion-positive CRC patient to regorafinib}

The absence of a concurrent driver mutation in RET fusion-positive CRC patients suggest that they may respond to a RET kinase inhibitor. Patient 1 was a 63-year-old woman who was diagnosed with stage IV sigmoid colon adenocarcinoma, and the PET/CT and MRI scans showed widespread metastases most notable for an enlarged liver that was nearly replaced by tumor (Figure 3A). Patient 1 declined chemotherapy-based treatment and wished to minimize therapy related toxicity. She was treated with low-dose regorafenib $80 \mathrm{mg}$ daily for 3 days and continued at $40 \mathrm{mg}$ daily. Clinically, patient $1 \mathrm{had}$ resolution of her early satiety and abdominal discomfort within 1 week of regorafenib initiation. She had a rapid CEA response from 471 to 158 and LDH response from 3310 to 1651 after 18 days of treatment (Figures 3B3C). No further follow-up was available as the patient succumbed to urosepsis shortly thereafter. Her clinical and CEA tumor marker responses suggested regorafenib has single agent activity in RET fusion positive CRC.

\begin{tabular}{|c|c|c|c|c|}
\hline \multirow{5}{*}{$A$} & \multirow{2}{*}{\multicolumn{2}{|c|}{ Genes }} & \multirow{2}{*}{ 5' Fusion Partner (n) } & \multirow[b]{2}{*}{ Mutations (\%) } \\
\hline & & & & \\
\hline & a & RET & (0) & $84 / 1,489(5.7 \%)$ \\
\hline & - & ALK & C2orf44; EML4 (3) & $118 / 1279(9.2 \%)$ \\
\hline & - & ROS & SLC34A2 (1) & $93 / 1243(7.5 \%)$ \\
\hline \multirow{12}{*}{$\operatorname{RAS}_{m u t}$} & $\overline{\mathbf{a}}$ & NTRK1 & TPM3 (2) & $46 / 1152(4.0 \%)$ \\
\hline & $\overline{\mathbf{a}}$ & NTRK3 & ETV6 (1) & $73 / 1374(5.3 \%)$ \\
\hline & a & $\mathrm{RSPO} 2$ & EIF3E (2) & $31 / 1063(2.9 \%)$ \\
\hline & a & $\mathrm{RSPO} 3$ & PTPRK (5) & $14 / 1063(1.3 \%)$ \\
\hline & $\overline{\mathbf{a}}$ & TCF7L2 & $V T I 1 A(4)$ & $101 / 1089(9.3 \%)$ \\
\hline & a & ERBB2 & (0) & $85 / 2,040(4.2 \%)$ \\
\hline & a & NRG1 & (0) & $58 / 1,064(5.5 \%)$ \\
\hline & - & PIKЗCA & (0) & $1,740 / 12,502(13.9 \%)$ \\
\hline & [1] & PTCH1 & (0) & $173 / 1147(15.1 \%)$ \\
\hline & a & BRAF & $(0)$ & $9,389 / 74,924(12.5 \%)$ \\
\hline & - & $B C L 11 B$ & (0) & $55 / 1089(5.1 \%)$ \\
\hline & a & KRAS & (0) & $18,378 / 53,364(34,4 \%)$ \\
\hline
\end{tabular}

B Colorectal Cancer Genomic Protocol

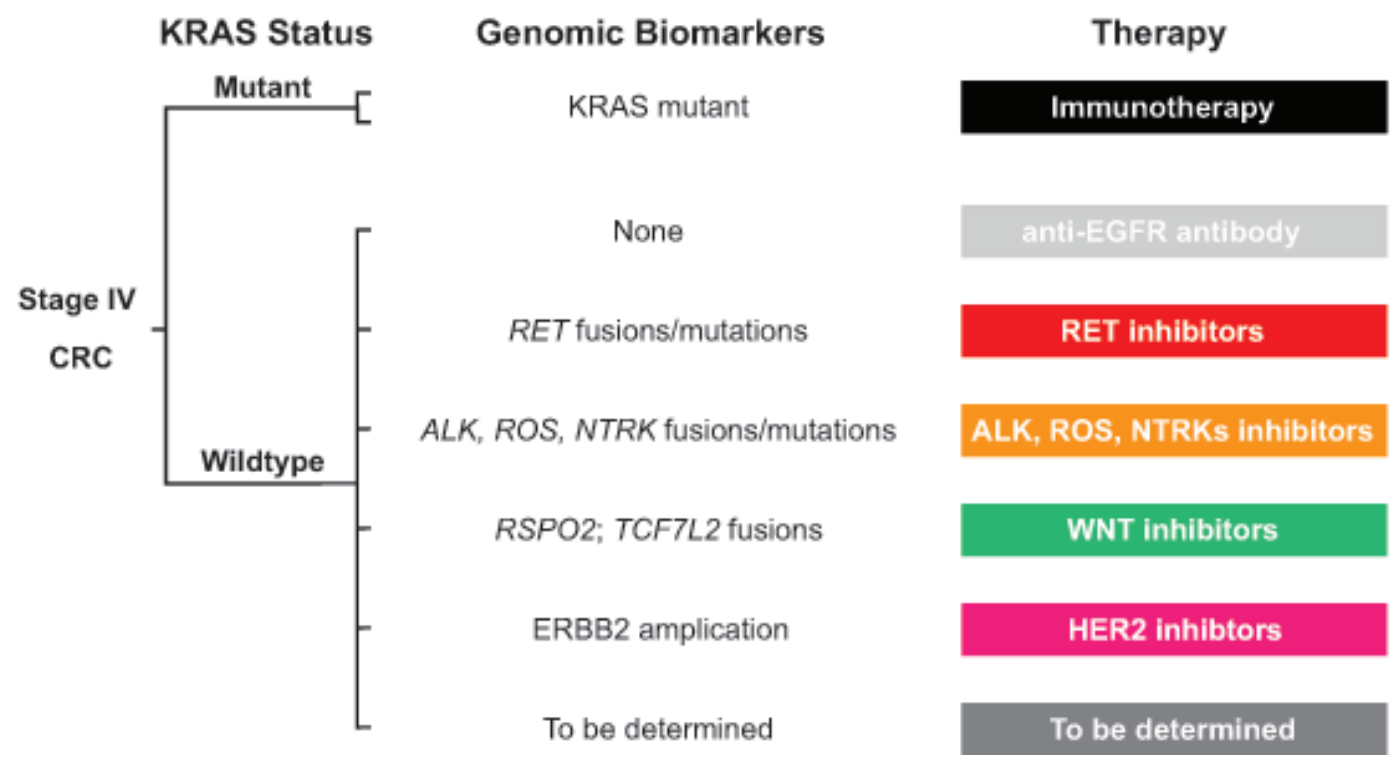

Figure 4: Colorectal cancer classification based on genomic biomarkers. A. Schematic classification of colorectal cancer based on genomic biomarkers with accompanying table showing fusion partner numbers (n) and gene point mutations available from the COSMIC database. B. Schematic of a Colorectal Cancer Genomic Protocol for stage IV CRC patients with assignment of therapy based on specific genomic biomarkers. 


\section{Colorectal cancer classification based on genomic biomarkers}

Next, we analyzed the Catalogue of Somatic Mutations in Cancer (COSMIC) database for cancerrelated gene fusions and point mutations in CRC patients. In addition to the six RET fusion kinases we have identified, there were other fusion kinases involving $A L K$, $N T R K$ and ROS and WNT pathway fusions involving RSPO and TCF7L2 (Figure 4A) [8, 17-21]. Based on the COSMIC database, genetic alterations by rearranged fusions were less common than missense or nonsense point mutations in those genes. RET point mutations occurred in $5.7 \%$ of CRC patients, and 30 of the 84 RET point mutations were localized within the RET kinase domain (Figure 4A). Notably, 8 RET mutations occurred specifically at E768, R844, S904, R912 and M918 (data not shown), which corresponded to hereditary RET mutated sites in familiar medullary thyroid carcinoma and multiple endocrine neoplasia type 2B [6,22].

\section{DISCUSSION}

Using comprehensive genomic profiling we have identified six RET fusion kinases in CRC and provide early clinicopathologic characteristics of this patient subset. The RET fusion kinase appears to be a mutually exclusive oncogenic driver that does not overlap with other known driver mutations such as $K R A S, B R A F$, $E G F R$ and PIK3CA and oncogenic fusion kinases involving $A L K$, ROSI and NTRK. Since we observed a low $0.2 \%$ frequency in unselected CRC patients, this mutual exclusivity may facilitate prospective screening for RET fusion kinase in CRC patients who are pan-negative for other known driver mutations. Further, the breakpoint locations observed on two of our patients appear to be novel. Our clinicopathologic observation that RET fusion kinase occurs in younger never smoker patients without other known driver alterations is consistent with observations in fusion kinase driven NSCLC and will require further validation in a larger cohort of CRC patients $[11,23]$.

Our data suggest that CRC patients who harbor RET fusion kinase without a concurrent driver mutation may respond to regorafenib, a potent RET kinase inhibitor with an $\mathrm{IC}_{50}$ of $\sim 1.5 \mathrm{n} \mathrm{M} \mathrm{[12].} \mathrm{The} \mathrm{in-vitro} \mathrm{studies} \mathrm{suggest} \mathrm{low}$ dose regorafenib potently suppresses RET fusion-positive cancer cells but has minimal inhibitory activity in RET fusion-negative CRC cells. Indeed, only $1 \%$ of unselected metastatic CRC patients treated with regorafenib had an objective or partial response in the CORRECT trial, which demonstrated a 1.4 month overall survival benefit compared to placebo [24]. We cannot preclude the possibility that the clinical activity of regorafenib in the CORRECT trial may be due to its inhibition of other kinase targets such as PDGFR or VEGFR, and RET fusion status analysis of the $1 \%$ regorafenib responders is needed. Since these RET fusion-positive CRC patients received a high median dose of regorafenib $147 \mathrm{mg}$ daily, additional potential off-target effects likely contributed to the 54\% incidence rate of severe or life-threatening adverse events [24]. Notably, RET fusion-positive cancer cells, but not $R E T$ fusion-negative cancer cells, had similar exquisite sensitivity to regorafenib, vandetanib and lenvatinib, which share RET and VEGFR kinases as the only common molecular targets. In light of the poor single agent activity of angiogenesis inhibitor in CRC patients, our findings suggest that the presence of RET fusion kinase identifies a subset of CRC patients with exceptional response to RET kinase inhibition. Further work and larger series are required to confirm and expand upon our findings.

Treatment of metastatic CRC patients with regorafenib at the maximum tolerated dose as used in the CORRECT trial has significant toxicity, and low-dose regorafenib may be of sufficient potency to inhibit tumors driven by RET fusion kinase [24]. To minimize therapy related toxicity and improve anti-RET kinase response, it is worthwhile to evaluate the therapeutic index of currently available anti-RET tyrosine kinase inhibitors in the subset of CRC patients who express RET fusion kinase. Although there may be a tissue-specific or contextual contribution to response, our findings are similar to reported responses to RET-directed therapies in thyroid cancer and NSCLC. Currently, several tyrosine kinase inhibitors with antiRET kinase activity such as vandetanib, lenvatinib, ponatinib, and carbozantinib are at various stages of clinical development for medullary thyroid carcinoma, in which $R E T$ is the critical oncogenic driver, and lung adenocarcinoma. Vandetanib, cabozantinib, and lenvatinib are FDA-approved as treatment in advanced thyroid cancer, since they significantly prolonged progressionfree survival when compared to placebo [14, 15, 25]. In RET-fusion positive NSCLC patients who had progressed on prior chemotherapy, treatment with the RET kinase inhibitors, vandetanib or carbozantinib, results with partial responses in three patients [26, 27]. We now extend the clinical activity of RET fusion kinase inhibition to CRC patients.

Our analysis of the COSMIC database summarizes the frequency of oncogenic fusion kinases and point mutations in CRC patients. However, the COSMIC database most likely underestimates the frequency of gene fusions and point mutations in CRC patients since the entire coding sequence of each gene was not determined. Using a comprehensive genomic profiling approach, we prospectively identified 6 RET fusions in $\mathrm{CRC}$ and an overall RET fusion frequency of $0.2 \%$. The identification of CRC patients with actionable RET fusion kinase provides further evidence of the impact that NGS has on clinical decision-making, and we anticipate the rapid adoption of prospective genomic profiling as a part 
of standard practice. The availability of NGS facilitates a therapeutic genomic paradigm to classify CRC based on actionable genomic biomarkers such as RET, ALK, NTRK, $R O S$ and $E R B B 2$, which may facilitate the clinical trial development of a Colorectal Cancer Genomic Protocol (Figure 4B).

\section{MATERIALS AND METHODS}

\section{Next generation sequencing}

Next-generation sequencing (NGS) assay covering 3,769 exons of 236 cancer-related genes and 47 introns of 19 genes frequently rearranged in cancer (Supplemental Table 1) was performed by Foundation Medicine, Inc., a CLIA-certified and CAP-accredited laboratory, based on a modified published protocol.[8] Briefly, formalin-fixed, paraffin-embedded (FFPE) specimen quality of volume $>1 \mathrm{~mm}^{3}$, nucleated cellularity $>80 \%$ or $>30,000$ cells, and $>20 \%$ tumor nuclei was ensured by macro-dissection as needed and confirmed by a pathologist. DNA was extracted using the Promega Maxwell 16 Tissue LEV DNA kit and quantified using an Invitrogen Picogreen fluorescence assay. Library Construction was performed with 50-200 ng of DNA sheared by sonication (Covaris E210) to $~ 100-400$ bp before end-repair, dA addition, ligation of indexed Illumina sequencing adaptors and PCR amplification for 10 cycles using Kapa HiFi. Solution phase hybridization was performed with a custom baitset of 120-bp biotinylated DNA oligonucleotides (Integrated DNA Technology), and 49 x 49 paired-end sequencing was performed using the Illumina HiSeq 2000 and Illumina HiSeq 2500 platforms. Sequence alignment, PCR duplicate read removal, and local alignment optimization was performed using BWA aligner 0.5.9, Picard 1.47 (http://picard.sourceforge.net/), Samtools 0.1.12a, and GATK 1.0.4705, and variant calling was performed using custom tools. Base substitutions were called using a Bayesian methodology, short indels were called using local assembly, copy number alterations were called through comparison to process-matched normal controls, and rearrangements were called using chimeric read pairs clustered by genomic position. Somatic variants were annotated using COSMIC and germline variants were removed using dbSNP.

\section{Patients, cell lines and reagents}

Informed consents for diagnostic testing and therapy were obtained from patients involved in this study. SW48, SW480 and SW620 cells were obtained from American Type Culture Collection (ATCC; Manassas, VA). Lc2/ ad cells were obtained from Sigma-Aldrich (St. Louis, MO). We transduced SW48 cells with pCCL-KRAS ${ }^{12 V}$ lentivirus to obtain SW48-KRAS ${ }^{12 \mathrm{~V}}$ cells. All cells were thawed from frozen stocks expanded from original cells obtained from ATCC and cultured for less than 3 months in Dulbecco's Modified Eagle Medium supplemented with $10 \%$ fetal bovine serum. Regorafenib, vandetanib and lenvatinib were obtained from Selleck pharmaceutical (Houston, TX).

\section{Quantitative PCR}

Purified mRNA from human tumor cells using a HiPure RNA isolation kit (Roche Life Science) were reverse transcribed with SuperScript Reverse Transcriptase II (Invitrogen) to generate cDNA template. Quantitative PCR was performed on the Roche Lightcycler 480 II with the addition of Taq polymerase, SYBR green (Roche Life Science) and primer pairs (Fisher Scientific). RET- forward primer (5'-GGCTTGTCCCGAGATGTTTA-3') and RET reverse primer ( 5 '-TCTTTTGGTGTCCTGCTGTG-3') recognized the $R E T$ tyrosine kinase domain, and CCDC6-197 forward primer (5'-TGCAGCAAGAGAACAAGGTG-3') and RET-2318 reverse primer (5'-CAGGCCCCATACAATTTGAT-3') flanked the CCDC6-RET fusion site.

\section{Cell toxicity assay}

A total of 8,000 cells/well were plated in $100 \mu$ of culture media in 96-well plates and treated the next day with $100 \mu \mathrm{l}$ of $0.1 \%$ DMSO media control or drugs at the indicated concentrations. After 72 hours, AlamarBlue (Life Technologies) was added, and cellular fluorescence was quantified per manufacturer instruction with a BioTEK Synergy 2 microplate reader.

\section{ACKNOWLEDGMENTS}

This study was supported by the American Cancer Society ACS-IRG 98-279-07 grant (to VKC) and the National Cancer Institute P30CA062203 grant. PJS, VAM, SMA, EMS, JSR, and SB are employed by and have equity interest in Foundation Medicine.

\section{CONFLICTS OF INTEREST}

The other authors have no potential conflicts of interest.

\section{REFERENCES}

1. Douillard JY, Oliner KS, Siena S, Tabernero J, Burkes R, Barugel M, Humblet Y, Bodoky G, Cunningham D, Jassem J, Rivera F, Kocakova I, Ruff P, Blasinska-Morawiec M, Smakal M, Canon JL, et al. Panitumumab-FOLFOX4 
treatment and RAS mutations in colorectal cancer. N Engl J Med. 2013; 369:1023-1034.

2. Comprehensive molecular characterization of human colon and rectal cancer. Nature. 2012; 487:330-337.

3. Krause DS and Van Etten RA. Tyrosine kinases as targets for cancer therapy. N Engl J Med. 2005; 353:172-187.

4. Druker BJ, Talpaz M, Resta DJ, Peng B, Buchdunger E, Ford JM, Lydon NB, Kantarjian H, Capdeville R, OhnoJones S and Sawyers CL. Efficacy and safety of a specific inhibitor of the BCR-ABL tyrosine kinase in chronic myeloid leukemia. N Engl J Med. 2001; 344:1031-1037.

5. Lynch TJ, Bell DW, Sordella R, Gurubhagavatula S, Okimoto RA, Brannigan BW, Harris PL, Haserlat SM, Supko JG, Haluska FG, Louis DN, Christiani DC, Settleman $J$ and Haber DA. Activating mutations in the epidermal growth factor receptor underlying responsiveness of non-small-cell lung cancer to gefitinib. N Engl J Med. 2004; 350:2129-2139.

6. Mulligan LM. RET revisited: expanding the oncogenic portfolio. Nat Rev Cancer. 2014; 14:173-186.

7. Takahashi M, Ritz J and Cooper GM. Activation of a novel human transforming gene, ret, by DNA rearrangement. Cell. 1985; 42:581-588.

8. Lipson D, Capelletti M, Yelensky R, Otto G, Parker A, Jarosz M, Curran JA, Balasubramanian S, Bloom T, Brennan KW, Donahue A, Downing SR, Frampton GM, Garcia L, Juhn F, Mitchell KC, et al. Identification of new ALK and RET gene fusions from colorectal and lung cancer biopsies. Nat Med. 2012; 18:382-384.

9. Takeuchi K, Soda M, Togashi Y, Suzuki R, Sakata S, Hatano S, Asaka R, Hamanaka W, Ninomiya H, Uehara H, Lim Choi Y, Satoh Y, Okumura S, Nakagawa K, Mano $\mathrm{H}$ and Ishikawa Y. RET, ROS1 and ALK fusions in lung cancer. Nat Med. 2012; 18:378-381.

10. Santoro M, Dathan NA, Berlingieri MT, Bongarzone I, Paulin C, Grieco M, Pierotti MA, Vecchio G and Fusco A. Molecular characterization of RET/PTC3; a novel rearranged version of the RETproto-oncogene in a human thyroid papillary carcinoma. Oncogene. 1994; 9:509-516.

11. Wang R, Hu H, Pan Y, Li Y, Ye T, Li C, Luo X, Wang L, Li H, Zhang Y, Li F, Lu Y, Lu Q, Xu J, Garfield D, Shen $\mathrm{L}$, et al. RET fusions define a unique molecular and clinicopathologic subtype of non-small-cell lung cancer. J Clin Oncol. 2012; 30:4352-4359.

12. Wilhelm SM, Dumas J, Adnane L, Lynch M, Carter CA, Schutz G, Thierauch KH and Zopf D. Regorafenib (BAY 73-4506): a new oral multikinase inhibitor of angiogenic, stromal and oncogenic receptor tyrosine kinases with potent preclinical antitumor activity. Int J Cancer. 2011; 129:245255.

13. Matsubara D, Kanai Y, Ishikawa S, Ohara S, Yoshimoto T, Sakatani T, Oguni S, Tamura T, Kataoka H, Endo S, Murakami Y, Aburatani H, Fukayama $M$ and Niki T. Identification of CCDC6-RET fusion in the human lung adenocarcinoma cell line, LC-2/ad. J Thorac Oncol. 2012; 7:1872-1876.

14. Wells SA, Jr., Robinson BG, Gagel RF, Dralle H, Fagin JA, Santoro M, Baudin E, Elisei R, Jarzab B, Vasselli JR, Read J, Langmuir P, Ryan AJ and Schlumberger MJ. Vandetanib in patients with locally advanced or metastatic medullary thyroid cancer: a randomized, double-blind phase III trial. J Clin Oncol. 2012; 30:134-141.

15. Schlumberger M, Tahara M, Wirth LJ, Robinson B, Brose MS, Elisei R, Habra MA, Newbold K, Shah MH, Hoff AO, Gianoukakis AG, Kiyota N, Taylor MH, Kim SB, Krzyzanowska MK, Dutcus CE, et al. Lenvatinib versus placebo in radioiodine-refractory thyroid cancer. N Engl J Med. 2015; 372:621-630.

16. Karapetis CS, Khambata-Ford S, Jonker DJ, O'Callaghan CJ, Tu D, Tebbutt NC, Simes RJ, Chalchal H, Shapiro JD, Robitaille S, Price TJ, Shepherd L, Au HJ, Langer C, Moore MJ and Zalcberg JR. K-ras mutations and benefit from cetuximab in advanced colorectal cancer. N Engl J Med. 2008; 359:1757-1765.

17. Lin E, Li L, Guan Y, Soriano R, Rivers CS, Mohan S, Pandita A, Tang J and Modrusan Z. Exon array profiling detects EML4-ALK fusion in breast, colorectal, and nonsmall cell lung cancers. Mol Cancer Res. 2009; 7:14661476.

18. Martin-Zanca D, Hughes SH and Barbacid M. A human oncogene formed by the fusion of truncated tropomyosin and protein tyrosine kinase sequences. Nature. 1986; 319:743-748.

19. Aisner DL, Nguyen TT, Paskulin DD, Le AT, Haney J, Schulte N, Chionh F, Hardingham J, Mariadason J, Tebbutt $\mathrm{N}$, Doebele RC, Weickhardt AJ and Varella-Garcia M. ROS1 and ALK fusions in colorectal cancer, with evidence of intratumoral heterogeneity for molecular drivers. Mol Cancer Res. 2014; 12:111-118.

20. Seshagiri S, Stawiski EW, Durinck S, Modrusan Z, Storm EE, Conboy CB, Chaudhuri S, Guan Y, Janakiraman V, Jaiswal BS, Guillory J, Ha C, Dijkgraaf GJ, Stinson J, Gnad F, Huntley MA, et al. Recurrent R-spondin fusions in colon cancer. Nature. 2012; 488:660-664.

21. Bass AJ, Lawrence MS, Brace LE, Ramos AH, Drier Y, Cibulskis K, Sougnez C, Voet D, Saksena G, Sivachenko A, Jing R, Parkin M, Pugh T, Verhaak RG, Stransky N, Boutin AT, et al. Genomic sequencing of colorectal adenocarcinomas identifies a recurrent VTI1A-TCF7L2 fusion. Nat Genet. 2011; 43:964-968.

22. Phay JE and Shah MH. Targeting RET receptor tyrosine kinase activation in cancer. Clin Cancer Res. 2010; 16:5936-5941.

23. Shaw AT, Yeap BY, Mino-Kenudson M, Digumarthy SR, Costa DB, Heist RS, Solomon B, Stubbs H, Admane S, McDermott U, Settleman J, Kobayashi S, Mark EJ, Rodig SJ, Chirieac LR, Kwak EL, et al. Clinical features and outcome of patients with non-small-cell lung cancer who harbor EML4-ALK. J Clin Oncol. 2009; 27:4247-4253. 
24. Grothey A, Van Cutsem E, Sobrero A, Siena S, Falcone A, Ychou M, Humblet Y, Bouche O, Mineur L, Barone C, Adenis A, Tabernero J, Yoshino T, Lenz HJ, Goldberg RM, Sargent DJ, et al. Regorafenib monotherapy for previously treated metastatic colorectal cancer (CORRECT): an international, multicentre, randomised, placebo-controlled, phase 3 trial. Lancet. 2013; 381:303-312.

25. Elisei R, Schlumberger MJ, Muller SP, Schoffski P, Brose MS, Shah MH, Licitra L, Jarzab B, Medvedev V, Kreissl MC, Niederle B, Cohen EE, Wirth LJ, Ali H, Hessel C, Yaron Y, et al. Cabozantinib in progressive medullary thyroid cancer. J Clin Oncol. 2013; 31:3639-3646.

26. Drilon A, Wang L, Hasanovic A, Suehara Y, Lipson D, Stephens P, Ross J, Miller V, Ginsberg M, Zakowski MF, Kris MG, Ladanyi M and Rizvi N. Response to Cabozantinib in patients with RET fusion-positive lung adenocarcinomas. Cancer Discov. 2013; 3:630-635.

27. Gautschi O, Zander T, Keller FA, Strobel K, Hirschmann A, Aebi S and Diebold J. A patient with lung adenocarcinoma and RET fusion treated with vandetanib. J Thorac Oncol. 2013; 8:e43-44. 